\title{
Quel enseignement de la lecture et de la littérature à l'heure des « compétences »?
}

\section{Jean-Louis Dufays}

\section{(2) OpenEdition}

\section{Journals}

\section{Édition électronique}

URL : http://journals.openedition.org/pratiques/1747

DOI : $10.4000 /$ pratiques. 1747

ISSN : 2425-2042

\section{Éditeur}

Centre de recherche sur les médiations (CREM)

\section{Édition imprimée}

Date de publication : 15 juin 2011

Pagination : 227-248

\section{Référence électronique}

Jean-Louis Dufays, «Quel enseignement de la lecture et de la littérature à l'heure des

" compétences »? », Pratiques [En ligne], 149-150 | 2011, mis en ligne le 17 juin 2014, consulté le 10 décembre 2020. URL : http://journals.openedition.org/pratiques/1747 ; DOI : https://doi.org/10.4000/ pratiques. 1747

\section{(c) Tous droits réservés}




\title{
Quel enseignement de la lecture et de la littérature à l'heure des « compétences ॥?
}

\author{
Jean-Louis Dufays
}

Université catholique de Louvain - IACCHOS - CRIPEDIS

Voilà deux décennies déjà que la notion de lecture littéraire s'est développée dans le champ de la théorie de la réception et un peu plus de quinze ans qu'elle a commencé à servir de modèle didactique, de pratique de référence pour l'enseignement-apprentissage de la lecture des textes littéraires - voire des textes en général - de la maternelle à l'université.

Parallèlement, la notion de compétence a été implantée peu ou prou dans les systèmes éducatifs de bon nombre de pays, dont elle a entrainé une transformation substantielle des programmes d'enseignement. Dans le cas de l'enseignement de la lecture et de la littérature, il en est résulté une importance accrue accordée aux productions des élèves, comprises non plus seulement comme des traces d'apprentissage en cours mais comme des performances complexes censées réinvestir un ensemble articulé de « ressources » situées dans un contexte communicatif «porteur de sens ». L'impératif des compétences se heurte cependant à une difficulté de principe : en matière de lecture et de littérature, l'évaluation des performances importe-t-elle davantage que l'apprentissage des savoirs, des savoir-faire et des attitudes qui permettront à l'élève de les réaliser ? Autrement dit, quelle est la pertinence de l'approche par compétence pour l'apprentissage de la lecture et de la littérature ? Et dans quelle mesure est-elle compatible avec le modèle didactique de la lecture littéraire ?

Le présent article tentera de répondre à ces questions en procédant en quatre temps. Il s'agira d'abord de faire le point (1) sur la notion de lecture littéraire, puis (2) sur celle de compétence, telle qu'elle est présentée par certains programmes d'enseignement, afin de dégager (3) ses implications sur la didactique de la littérature et ses articulations éventuelles avec le modèle de la lecture littéraire. Je m'interrogerai ensuite (4) sur la place de l'approche par compétence dans les pratiques effectives d'enseignement de la lecture et de la littérature avant de tirer enfin un bilan critique de ses apports et de ses limites. 


\section{Le point sur la lecture littéraire}

\subsection{Origines et évolution de la notion}

Il n'est peut-être pas inutile de rappeler que le modèle didactique de la lecture littéraire a été développé dans les années 1980 dans la foulée de l'avènement des théories de la réception, qui résultait lui-même de l'épuisement supposé des paradigmes centrés sur l'auteur et sur le texte. Suite logique des manifestes relativistes de Nietzsche et de Dilthey, puis des prises de positions de Sartre, de Gadamer et d'Ingarden sur les pouvoirs et la responsabilité du lecteur, puis des mutations culturelles des années 1960, l'intérêt porté à l'activité du récepteur s'est épanoui dans l'Esthétique de la réception dans les années 1970-80, et il a abouti à un déplacement de perspective fondamental : désormais la source de l'effet littéraire n'était plus étudié au départ de l'œuvre mais au départ de l'activité du lecteur.

Rien d'étonnant à ce que, dans un tel contexte, en réplique à la notion d'écriture littéraire, on ait vu se développer son pendant, la lecture littéraire. Cette notion apparait déjà chez Blanchot (1955 : 258-261), mais celui-ci la définit comme un acte fictif, une pure instance idéale, qui s'oppose au lecteur effectif de l'espace public : la lecture littéraire blanchotienne est une exigence adressée au lecteur, une condition de possibilité de l'illusion proposée par le monde littéraire. Marghescou (1974/2009) adoptera une position plus empirique en opposant le « régime de lecture littéraire », fondé sur la suspension de la portée référentielle du signe et sur l'activation de ses potentialités symboliques plurielles, au « régime de lecture référentielle », qui se contente quant à lui de décoder les anecdotes en privilégiant la dénotation. Cette distinction sera reprise par Gervais (1993), qui définira la lecture littéraire comme le passage de la régie linéaire et impliquée de la progression à la régie tabulaire et distanciée de la compréhension. De Certeau (1980) quant à lui ne parle pas de lecture «littéraire », mais de lecture comme «braconnage », qu'il définit comme le lieu de l'émancipation du lecteur, comme l'expression de sa créativité dans les marges des sens présentés comme légitimes. Le grand théoricien de la lecture littéraire est Picard (1986), qui réconcilie les tenants de la lecture référentielle et ceux de la lecture savante en définissant la « lecture littéraire » comme une combinaison d'une part de trois instances : celles du liseur, lié au corps du lecteur, du $l u$, dominé par les référents du texte, et du lectant, qui au contraire les domine, et d'autre part de trois évaluations dialectiques, qui valorisent tour à tour le pluriel du sens et sa cohérence (sens vs signification), la véracité des données et leur caractère fictionnel (vérité $v s$ mensonge), leur portée modélisante et leur dimension subversive (conformité $v s$ subversion). Pour Picard (1986 : 266), la lecture littéraire est à la fois «subversion dans la conformité » (jeu avec les conventions, les genres), «élection du sens dans la polysémie » (jeu avec le(s) sens) et " modélisation par une expérience de réalité fictive » (jeu avec les représentations du monde).

A travers ces différentes élaborations théoriques, on voit se dessiner trois orientations distinctes, qui constituent autant de modèles didactiques de l'activité interprétative :

$1^{\circ}$ L'option de Marghescou, qu'on peut comparer notamment à celles de Riffa- 
terre (1979) et de Gervais (1992), privilégie la distanciation, l'écart esthétique, le symbole, la polysémie. Le modèle qui sert ici de référence est l'activité d'un lecteur « lettré », formé, savant.

$2^{\circ}$ L'option de Michel de Certeau, qu'on retrouve dans une certaine mesure dans l'éloge de l'étonnement de Poslaniec (2002) et dans l'éloge de la paraphrase de Daunay (2002), privilégie au contraire la participation du lecteur " ordinaire », sa lecture « immédiate » du sens « premier», mais aussi les voies insoupçonnées par lesquelles il s'approprie les textes en «braconnant» du sens d'une manière personnelle, sans se laisser enfermer dans les normes de la lecture légitime. Le modèle retenu ici est l'activité quotidienne des lecteurs réels dont on cherche à valider et à systématiser les processus.

$3^{\circ}$ Picard enfin, et à sa suite Jouve (1993) et votre serviteur (Dufays, 1994/2010a), considèrent que la lecture littéraire réside dans la tension dialectique entre les deux modes précités. Pour ces auteurs, lire littérairement revient à optimiser le fonctionnement tendanciel de toute lecture en alternant sciemment les postures du lu ou du lisant et du lectant (Picard et Jouve), de la participation et de la distanciation (Dufays), et en oscillant entre le « colmatage des blancs » (cher à Iser, 1985) et la « lecture suspensive» (préconisée par Steinmetz, 1981), entre la lecture " quasi-pragmatique » et la lecture "pseudo-référentielle » (Stierle, 1979) $^{(1)}$.

L'objectivité oblige à reconnaitre qu'entre ces différentes conceptions le débat n'est pas clos, et que le terme de lecture littéraire sert aujourd'hui encore à désigner des processus parfois bien différents. Le point commun est l'idée d'une lecture exigeante, dont le lecteur assume consciemment les choix et les processus. Par delà leurs oppositions, les trois conceptions précitées s'accordent en effet pour attribuer au lecteur la responsabilité de sa lecture et pour considérer que la littérarité se construit au moins partiellement dans celle-ci.

\subsection{Et le sujet lecteur ?}

Parallèlement à la lecture littéraire - ou plus exactement en réaction contre la conception rationnelle et distanciée de celle-ci -, certains didacticiens (Rouxel \& Langlade, 2004) ont développé au cours des années 2000 la notion de «sujet lecteur ». Dans la lignée des travaux de Michel de Certeau et de certains psychanalystes (Bellemin-Noël notamment), celle-ci témoigne d'un intérêt qui parait $a$ priori salutaire à l'égard de l'élève comme sujet singulier, de son épanouissement, de sa liberté, de son pouvoir. Elle comporte cependant deux limites.

La première tient à l'ambiguïté du « sujet» dont il est question : s'agit-il du sujet effectif ou d'un sujet « idéal »? A lire les travaux - généralement très subtils qui décrivent l'activité du sujet lecteur, on a plutôt l'impression qu'il s'agit d'un lecteur d'exception, capable de déployer une grande variété de connaissances, de valeurs et de stratégies qu' on chercherait en vain dans la pratique effective de la plupart des jeunes lecteurs, a fortiori en contexte scolaire.

(1) Pour une synthèse de ces différentes approches, voir Dufays, Gemenne et Ledur, 2005 : 87 97 et Dufays, 2010:155-160. Pour une mise en perspective avec les notions de lectures " méthodique ", " cursive » et " analytique », voir les mises au point éclairantes de Raymond Michel (1998, 2001). 
En second lieu, si le «sujet lecteur » sert à désigner les fonctionnements subjectifs observables du lecteur réel, ne faudrait-il pas alors se rappeler que, comme le disait Barthes, la subjectivité d'un lecteur est faite en grande partie de stéréotypes dont il ne perçoit pas qu'il les partage avec toute une communauté $^{(2)}$ ? Au-delà de son individualité, l'élève est aussi membre d'une communauté - discursive et interprétative ( $c f$. Bernié, 2002) -, qui partage certains codes et modes de lecture qu'il importe de reconnaitre et de stabiliser, non seulement afin de garantir une certaine pérennité du lien social (ce qui est une justification éthique déjà fondamentale), mais aussi, plus simplement, parce que le langage est un code collectif, dont les signes seraient dénués de sens s'ils n'étaient pas rattachés aux conventions d'une culture partagée. Dans Ecole et culture (1989), Jean-Claude Forquin dénonçait déjà les impasses d'une individualisation sans borne, ou bornée seulement par la culture mass-médiatique. On voit à ce propos que le paradigme de la lecture littéraire comme cadre descriptif - et non normatif - du caractère dialectique de toute lecture présente l'avantage de constituer un garde-fou tant contre les dérives de la subjectivité (qui peut parfois devenir délirante) que contre celles de la neutralité (qui peut parfois s'avérer desséchante). Le modèle didactique de la lecture littéraire comme va-et-vient n'exclut pas le sujet lecteur, mais il lui assigne une place sans lui être subordonné car il s'agit d'un paradigme plus puissant, qui implique à la fois l'activité subjective et l'activité intersubjective.

Une chose est sûre : au-delà de ses variantes, la notion de lecture littéraire a connu en peu de temps une évolution spectaculaire. Indépendamment des accentuations diverses dont elle a fait l'objet, cette notion a clairement migré de la théorie littéraire vers la didactique, au point d'occuper aujourd'hui une place au moins aussi visible dans le discours scolaire des pays francophones - surtout en France à l'école primaire et en Belgique au lycée - que dans la théorie littéraire elle-même.

\subsection{Difficultés et enjeux de la lecture littéraire en contexte didactique}

Pour autant, la mise en œuvre d'une didactique de la lecture littéraire n'est pas allée sans difficultés. Celles-ci tiennent d'abord au fait que cette notion est utilisée dans nombre de discours comme une pratique "naturalisée » au départ de théories savantes, mais dont la modélisation didactique reste floue, mal définie. Quand on voit l'usage très large qui en est fait et l'absence de réflexivité qui le caractérise le plus souvent, force est de constater que la seule référence à la lecture littéraire ne garantit aucune démarche spécifique. Chez beaucoup d'auteurs, le lexème « lecture littéraire » sert à désigner une notion molle, " passe partout », applicable à n'importe quelle lecture d'un texte littéraire. A l'inverse, lorsqu'elle revêt un sens normatif, cette pratique peut apparaitre comme trop exigeante, voire élitiste, solidaire des valeurs d'une classe : c'est que lui reproche Bertrand Daunay (1999), qui s'en prend à Picard, non pas en raison de sa défini-

(2) «La subjectivité est une image pleine, dont on suppose que j'encombre le texte, mais dont la plénitude, truquée, n'est que le sillage de tous les codes qui me font, en sorte que ma subjectivité a finalement la généralité même des stéréotypes. » (Barthes, 1970 : 16-17). 
tion de la lecture littéraire mais en raison des exemples et des commentaires qui lui servent à étayer celle-ci.

Ces difficultés, qui sont, à vrai dire, le lot de beaucoup de notions des sciences humaines, ne suffisent cependant pas à justifier l'évacuation de la « lecture littéraire »; elles invitent plutôt les didacticiens à se montrer vigilants et rigoureux lorsqu'ils y font référence. Elles les incitent, en premier lieu, à renoncer à faire de cette notion un simple synonyme de « lecture de la littérature », c'est-à-dire à dépasser une conception purement nominaliste, qui n'accorde à la lecture littéraire ni traits ni pouvoir spécifique. Les deux autres conceptions dominantes - la lecture littéraire comme distanciation objectivante ancrée dans un travail d'interprétation et de symbolisation ou comme activité subjective liée à l'usage référentiel de la langue - sont à prendre, quant à elles, comme deux états historiques de cette notion, qui ont prévalu l'une et l'autre un certain temps dans un certain nombre de discours, mais sont aujourd'hui dépassées par le paradigme du va-etvient qui apparait comme plus intégrateur, et partant plus puissant didactiquement. Cette supériorité factuelle de la conception dialectique, insistons-y, tient au fait qu'elle se présente moins comme la prescription artificielle d'une lecture idéale que comme le cadre descriptif permettant de comprendre le mouvement oscillatoire qui se jouerait dans toute lecture, si simple ou ordinaire qu'elle paraisse. Modèle de compréhension des lectures plutôt qu'emblème d'une lecture modèle, la notion de lecture littéraire invite à combiner sans les hiérarchiser les deux usages fondamentaux auxquels se prête de facto tout texte littéraire - voire, si on le veut bien, tout objet « attentionnel » (Genette 1997) -, à savoir l'usage « référentiel » et l'usage « poétique».

Mais si cette conception dialectique de la lecture littéraire parait susceptible de tenir la route en tant que modèle didactique, c'est avant tout parce qu'elle présente des enjeux didactiques évidents. Du côté de la participation, les enseignants ont tout intérêt à proposer à leurs élèves des activités comme le journal de lecture, la présentation de lectures « coups de cœur» ou la réécriture créative qui favorisent le rapport affectif et imaginaire aux textes et l'appropriation subjective de la littérature. Et du côté de la distanciation, on perçoit bien l'importance de développer des activités d'analyse adaptées à la diversité et à la complexité des textes, de familiariser les élèves avec les déterminations socioculturelles et les institutions de la littérature, de susciter une réflexion «méta » sur le fait littéraire et sur les différentes approches qu'il permet, tout en transmettant des références culturelles nécessaires à son intelligence historique ${ }^{(3)}$.

Il reste que, si elle parait appelée à servir de cadre pour la programmation des activités d'enseignement-apprentissage, la notion de lecture littéraire se heurte inévitablement à un autre paradigme dominant, mais transversal celui-là, celui de l'approche par compétence. La question qui se pose dès lors est celle de la compatibilité et des articulations possibles entre ces deux modèles.

(3) Ces différentes activités sont développées et illustrées tout au long de la $4{ }^{\mathrm{e}}$ partie du livre Pour une lecture littéraire (Dufays, Gemenne \& Ledur, 2005 : 163-337). 


\section{Le point sur l'approche par compétence}

\subsection{Six caractéristiques de la compétence}

Faut-il le rappeler, c'est à la fin du $\mathrm{XX}^{\mathrm{e}}$ siècle que la logique des compétences a déferlé dans le système éducatif de bon nombre de pays développés, dont elle a profondément modifié les objectifs et les contenus. Ce n'est pas le lieu ici de retracer par le détail l'historique de cette notion et des déplacements qu'elle a subis au cours de sa courte histoire, mais je crois utile d'en dégager quelques traits saillants.

Prenons pour point de départ la définition officielle de la compétence qui figure dans le décret « Missions » publiée par la Communauté française de Belgique en 1997 : la compétence est une « aptitude à mettre en œuvre un ensemble organisé de savoirs, de savoir-faire et d'attitudes permettant d'accomplir un certain nombre de tâches » ${ }^{(4)}$.

Comme l'a bien noté Marc Romainville (2001), sous ses dehors de simplicité, cette définition est en réalité lourde d'implications didactiques, qui peuvent apparaitre sinon en rupture, du moins en contraste avec les conceptions de l'enseignement-apprentissage qui avaient dominé jusque là. Six points sont en effet à relever :

$1^{\circ}$ La compétence est une habileté plurielle, complexe, qui excède le simple savoir-faire, car elle repose sur l'aptitude à mobiliser plusieurs apprentissages, les uns de type «déclaratif» (des savoirs), les autres de type «procéduraux » (des savoir-faire).

$2^{\circ}$ L'enseignant n'accède pas directement aux compétences de l'élève, mais s'en fait seulement une idée au travers des performances, c'est-à-dire des productions, forcément écrites ou orales, que celui-ci réalise. Autrement dit, évaluer une compétence revient à vérifier si l'élève peut accomplir des tâches relatives à celle-ci.

$3^{\circ}$ Dans l'approche par compétence, ni les savoirs ni les savoir-faire ne constituent donc plus des fins en soi : c'est leur valeur d'usage qui devient déterminante. Certains partisans de cette approche sont allés jusqu'à qualifier de «morts» ou d'inertes les savoirs non associés à l'usage d'une compétence. Audelà de son outrance, cette affirmation indique clairement que la focalisation sur les compétences des élèves privilégie non pas les processus de leur apprentissage ni les ressources mobilisées dans ce cadre, mais leurs résultats. L'approche par compétence participe de ce qu'on appelle les « learning outcomes», elle est centrée non pas sur la didactisation des contenus d'apprentissage mais sur leur finalisation (Romainville 2001). En favorisant une conception «actionnelle» de l'enseignement-apprentissage, elle renoue avec une forme de behaviourisme où l'output a priorité sur l'input, la fin sur les moyens, le produit sur le processus, le résultat sur la mise en œuvre.

(4) Mon école, comme je la veux! Ses missions. Mes droits et mes devoirs. Décret définissant les Missions prioritaires de l'Enseignement Fondamental et de l'Enseignement Secondaire et organisant les Structures propres à les atteindre, Bruxelles, Cabinet de la Ministre de 1'Education, 1997, p. 5, article 5. 
$4^{\circ}$ Selon les tenants de cette approche, les tâches ne reflètent des compétences que si elles sont non seulement globales et complexes, mais aussi « porteuses de sens », "significatives ». C'est ainsi que, dans un programme belge actuel du cours de français, chacune des six « compétences » prescrites pour chaque degré d'enseignement est présentée comme inscrite « dans une situation-problème significative $\gg{ }^{(5)}$.

$5^{\circ} \mathrm{Si}$ la compétence est une habileté complexe, les tâches qui la révèlent se doivent elles aussi d'être complexes. Etre compétent ne consiste pas à répondre à des questions élémentaires ou à effectuer des tâches mécaniques, mais à résoudre des problèmes. L'approche par compétence est ainsi souvent assimilée à ce qu'on appelle l'approche par problème ou APP. Plus précisément, pour pouvoir révéler une compétence, une situation d'évaluation se doit toujours d'être à la fois nouvelle et proche de celles proposées pendant l'apprentissage.

$6^{\circ}$ Parce que l'approche par compétence est dès lors indissociable de celle de tâche ou de situation, promouvoir une telle approche implique de déterminer les « familles de tâches » ou de « situations » qui permettront de manifester, et partant d'évaluer les compétences. Ces «familles » sont des ensembles de situations ou de tâches différentes entre elles, mais proches dans leurs paramètres, parmi lesquels il s'agit de puiser pour pouvoir évaluer si un élève est compétent.

\subsection{L'exemple des programmes belges de l'enseignement secondaire de transition}

Parmi les pays francophones, c'est en Communauté française de Belgique et au Québec que l'approche par compétence a été le plus profondément inscrite dans les systèmes éducatifs.

Certes, la France et la Suisse romande ont connu eux aussi, leur « vogue des compétences », mais du côté Suisse, la situation est restée assez éclatée en raison du caractère cantonal des programmes scolaires, et en France, même si certains chercheurs ont été pionniers en la matière (CEPEC, 1992) et même si on y a instauré la pratique des « carnets de compétence » dans l'enseignement primaire, la notion de compétence n'a pas été généralisée explicitement dans les programmes du collège et du lycée.

En Communauté française de Belgique, en revanche, sans doute en raison de la présence à cet endroit de chercheurs fortement impliqués dans l'approche par compétence (De Ketele, Roegiers, Paquay, Crahay ${ }^{(6)}$ ), l'approche par compétence a été instaurée de manière très explicite comme approche de référence de toutes les disciplines par le décret « Missions » de 1997, dont l'article 8 était formulé comme suit :

«Pour atteindre les objectifs généraux visés à l'article 6, les savoirs et les savoir-

(5) Programme Français $2^{e}$ degré et $3^{\mathrm{e}}$ degré Humanités générales et technologiques, Bruxelles, Fédération de 1'enseignement secondaire catholique, 2000-2002.

(6) Après avoir été l'un de ses propagateurs les plus vigoureux, ce dernier a toutefois pris fortement ses distances par la suite à l'égard de l'approche par compétences, dont il a dénoncé les dérives et qu'il considère aujourd'hui comme une approche qu'il convient de combiner avec d'autres ( $c f$. Crahay, 2006). 
faire, qu'ils soient construits par les élèves eux-mêmes ou qu'ils soient transmis, sont placés dans la perspective de l'acquisition de compétences. » ${ }^{(7)}$

Une ambiguïté se produit cependant d'emblée en ce qui concerne la relation entre compétence et savoir, car, si la compétence est définie comme une notion qui domine celle de savoirs et de savoir-faire (ceux-ci sont «placés dans la perspective de l'acquisition de compétences »), dans l'article 2 du même texte, les deux notions sont au contraire juxtaposées, comme si elles étaient sur un pied d'égalité : le deuxième objectif assigné à l'enseignement dans son ensemble est en effet d' " amener tous les élèves à s' " approprier des savoirs et à acquérir des compétences qui les rendent aptes à apprendre toute leur vie et à prendre une place active dans la vie économique, sociale et culturelle ».

Ce brouillage de la compétence va se confirmer dans les référentiels inter-réseaux qui seront publiés en 1999 dans la foulée du décret, avec pour fin de définir les compétences à atteindre tout au long de la scolarité. D'une part, que ce soit dans les Socles de compétence, qui fixent les objectifs de l'Enseignement fondamental et du premier degré du secondaire, ou dans les Compétences terminales, qui fixent les objectifs de l'Enseignement secondaire de manière transversale (pour les humanités techniques et professionnelles) ou pour chacune des disciplines (pour les humanités générales et technologiques), la notion de compétence est utilisée dans un sens qui ne la distingue guère de ce qu'on appelle ailleurs des savoir-faire. D'autre part, la relation des compétences avec les savoirs reste l'objet d'une hésitation manifeste puisque, alors que la compétence reste présentée comme la notion structurante qui domine celle des « savoirs ", l'un des référentiels juxtapose les deux termes (Compétences terminales et savoirs requis en...)

Mais si les référentiels dits « de compétences » ont donc renoncé, paradoxalement, à définir de réelles compétences, il n'en va pas de même des programmes rédigés par les deux grands réseaux d'enseignement ${ }^{(8)}$ pour la discipline « français ». A titre d'exemple, considérons ceux qui concernent les quatre dernières années de l'enseignement secondaire de transition : tous deux se sont attachés à définir pour chacun des deux « degrés » (ou cycles de deux ans) concernés, l'un quatre à six « fiches compétences », l'autre dix à onze « tâches-problèmes », qui sont autant d'activités complexes requérant la mobilisation d'un ensemble de savoirs et de savoir-faire, voire de savoir-être. Examinons ceci de plus près.

a) Dans le programme du réseau de la Communauté ${ }^{(9)}$, sur les trois tâchesproblèmes propres aux élèves du $2^{\mathrm{e}}$ degré du secondaire, une concerne la lecture et/ou la littérature : [1] «Présenter oralement, aux élèves de sa classe, un avis ar-

(7) Op. cit., p. 7.

(8) Pour rappel, l'enseignement belge est organisé par différents réseaux, tous subventionnés par l'Etat mais qui disposent d'une relative autonomie pour gérer leurs établissements et pour définir leurs programmes. Parmi eux, les deux principaux sont le réseau de la Communauté, qui dépend du Ministre de l'Education et gère environ $40 \%$ des écoles, et le réseau libre catholique, qui dépend de la Fédération de l'enseignement catholique ou Fésec et gère environ $50 \%$ des écoles.

(9) Ministère de la Communauté française, Enseignement secondaire générale et technique de transition $2^{e}$ et $3^{e}$ degrés. Programme d'études du cours de français, Bruxelles, AGERS, 2000. 
gumenté sur un récit ». Trois autres tâches littéraires figurent parmi les sept qui sont communes au $2^{\mathrm{e}}$ et au $3^{\mathrm{e}}$ degrés : [2] «Classer diverses espèces de récits et défendre oralement son classement», [3] «Enoncer, par écrit, à destination du professeur, ou oralement, à destination de l'ensemble de la classe, une comparaison portant sur deux ou plusieurs œuvres ou productions culturelles traitant soit de sujets identiques ou voisins, soit de sujets radicalement différents » et [4] «Présenter oralement un essai au professeur et/ou aux élèves de sa classe».

Enfin, on trouve quatre tâches de nature littéraire parmi les cinq tâches propres au $3^{\mathrm{e}}$ degré :

[5] «Rédiger et présenter oralement la synthèse d'un dossier portant sur...

- la vie culturelle à une époque affectée par des changements profonds (p. ex. ceux provoqués par l'esprit de la Renaissance...),

- un mouvement artistique international (p. ex. le romantisme...),

- un genre littéraire dans une ère culturelle ou dans un mouvement artistique (p. ex. la tragédie classique...),

- l'évolution d'un genre littéraire ou d'une subdivision d'un genre littéraire (p. ex. la comédie...),

- les avatars d'une tendance artistique (p. ex. le réalisme - ou l'idéalisme - dans la peinture et la littérature du XII ${ }^{\mathrm{e}}$ au XIX ${ }^{\mathrm{e}}$ siècle...),

- les avatars d'un mythe (p. ex. Caïn, Edipe...) ou d'un type littéraire (p. ex. l'artiste, l'aventurier...),

- une œuvre littéraire, philosophique ou scientifique (sciences humaines) de rupture (p. ex. Essais, Don Quichotte...).»

[6] « Appréhender un courant littéraire et artistique, le baroque [présenté à titre d'exemple]»

[7] « S'informer réciproquement, par des moyens divers, sur l'un...

- des courants d'idées (l'humanisme, la pensée des Lumières),

- des mouvements ou des écoles artistiques (le romantisme, le réalisme, le symbolisme, le surréalisme),

- des concepts fédérateurs (le baroque, le classicisme, la modernité, la culture contemporaine) figurant dans la partie « savoirs disciplinaires » du référentiel »

[8] « Rédiger, d'après un modèle donné, une présentation d'un roman destinée au professeur et répondre aux questions de ce dernier relatives à cette présentation »

On le voit, la logique de ces différentes tâches est avant tout actionnelle : à aucun moment ou presque il n'est question de cours magistral ni de connaissance directement transmise par le professeur qu'il s'agirait de restituer ; en permanence, les élèves sont mis en situation de recherche, d'activité autonome, de synthèse écrite ou d'exposé oral. Plus précisément, sur les huit tâches proposées, une part égale est consacrée aux exposés oraux - qui voient ainsi leur place s'accroitre considérablement - et aux présentations écrites. Trois portent sur la présentation d'une œuvre $(1,4,8)$, deux sur la comparaison de plusieurs œuvres et sur la présentation d'un dossier les concernant (courant, genre ou groupement thématique...) $(2,3,5,6,7)$, et presque toutes privilégient la recherche documentaire, qui devient ainsi l'activité de base du cours de français.

Autre constat : par le caractère très explicite et détaillé de ses consignes, ce 
programme présente une allure assez prescriptive, et les tâches assument leur caractère de "genres scolaires » : il est peu question ici de mimer des «pratiques sociales de référence ». Enfin, aucune allusion n'est faite, ni dans la présentation des activités, ni même dans la bibliographie générale proposée en appoint, à la notion de lecture littéraire, qui avait pourtant déjà le vent en poupe à l'époque où le programme a été rédigé, et les activités proposées ne sont guère placées sous le signe d'une oscillation entre des postures de lecture complémentaires.

On voit ainsi se dessiner une première conception de l'approche par compétence, qui se tient à l'écart tout à la fois de la lecture littéraire, des pratiques sociales extrascolaires et de l'autonomie accordée aux enseignants : même s'il n'est question que de mettre les élèves en activité, tout, ici, est prescrit avec précision, dans une perspective qui vise certes à former au mieux la culture et les gouts de l'élève mais relève moins, dans ses formes, de la réception littéraire que d'une activité scolaire assumée comme telle.

b) Dans le programme du réseau catholique ${ }^{(10)}$, l'approche par compétence se traduit tout autrement : plutôt que de présenter par le détail une diversité de « tâches-problèmes » articulant certains savoirs à certains savoir-faire, ce réseau a choisi de prescrire à chaque degré quatre à six « compétences d'intégration » formulées de manière synthétique en quelques lignes, et suivies d'une "fiche» de deux à quatre pages qui détaille les « objets à produire », les « apprentissages », les « contextes qui donnent du sens », des « conseils méthodologiques », et enfin un lexique et une bibliographie.

Le $3^{\text {e }}$ degré de transition (élèves de 16 et 17 ans) se voit ainsi attribuer les six compétences suivantes :

1. Lire le texte polémique et écrire le texte argumenté

2. Conduire une recherche documentaire et rédiger une synthèse de textes

3. Produire un exposé

4. Prendre sa place dans une discussion de groupe et rédiger un compte rendu

5. Lire des textes littéraires

6. Construire la notion de littérature

Parmi celles-ci, les trois premières, qui rappellent certaines des tâches qu'on vient de voir à l'œuvre dans le programme du réseau de la Communauté, peuvent fort bien se combiner à d' autres et concerner des textes littéraires. Quant à la $5^{\mathrm{e}}$ et la $6^{\mathrm{e}}$, qui concernent plus spécialement la lecture et la littérature, elles sont libellées comme suit :

[5] Dans une situation-problème significative, construire un ou plusieurs réseaux de signification, pour répondre à des questions suscitées par la lecture d'un texte, porter une appréciation personnelle sur le texte, faire part de son interprétation à travers divers moyens d'expression (au cours d'une discussion, dans un compte rendu de lecture, par des réécritures, des mises en voix ${ }^{(11)}$.

(10) Programme Français $2^{e}$ degré et $3^{\mathrm{e}}$ degré, op. cit.

(11) Cette compétence est pratiquement identique à celle qui est énoncée pour le $2^{\mathrm{e}}$ degré de transition et de technique de qualification du même réseau : " Dans une situation-problème significative, comprendre et interpréter un texte littéraire, porter une appréciation personnelle sur le texte et faire part de sa lecture par divers moyens d'expression». 
[6] Dans des situations-problèmes significatives, participer de manière réfléchie à la vie culturelle et élargir le champ de ses pratiques culturelles en abordant le concept de littérature sous divers éclairages croisés qui permettent d'en construire une définition complexe.

On le voit, lecture et littérature font l'objet de deux compétences distinctes : d'un côté, il s'agit d'apprendre à mieux lire, de l'autre, il s'agit d'apprendre à réfléchir à la notion de littérature. Par ailleurs, à la notion de "tâche-problème ", qui mettait l'accent sur le rôle du maitre, ce programme préfère celle de « situation-problème ", qui met davantage en évidence le caractère collectif et potentiellement négocié des activités, et plutôt que de détailler ces dernières, il se limite à en donner quelques exemples en quelques lignes. Ainsi, pour la compétence $\mathrm{n}^{\circ} 5$, quatre situations-problèmes sont proposées :

« [1] Dans le cadre d'un concours ou de l'attribution d'un prix littéraire (Prix des lycéens), les élèves sont invités à lire un ou plusieurs livres pour

- écrire une correspondance entre personnages, entre l'auteur et un personnage, entre le lecteur et l'auteur

- rapporter un entretien avec un personnage

- susciter des dialogues imaginaires entre auteurs, entre personnages littéraires

- créer des affiches de théâtre ou de cinéma

- rédiger des conseils de lecture pour un pairs ou

- débattre de leurs interprétations respectives et émettre une appréciation sur les livres lus.

[2] En préparation à la découverte d'une mise en scène (au théâtre ou sur vidéo) d'une pièce travaillée en classe, les élèves sont invités à imaginer, par équipes, une mise en scène ou une lecture-spectacle d'un extrait de la pièce ; ils présentent leur production à la classe et comparent leurs lectures et leurs choix.

[3] Dans le cadre d'une rencontre avec les parents ou avec d'autres classes, les élèves préparent un récital de poèmes qu'ils ont analysés et mémorisés.

[4] Dans le cadre de la lecture d'un roman, les élèves rédigent un journal personnel dans lequel ils consignent quelques traces de lecture où ils font part de leurs réactions et questionnements, en articulant diverses pratiques de lecture (investissement psycho-affectif, contexte et mode personnels de lecture, construction active de significations...). Le journal peut servir de tremplin à un échange d'opinions livrant les interprétations et les appréciations personnelles de l'œuvre lue » (pp. 26-27).

Attribution d'un prix, préparation d'un spectacle ou d'un récital, rédaction d'un journal personnel : par contraste avec le programme du réseau de la Communauté, on voit ici s'afficher nettement le souci d'ancrer les productions dans des pratiques sociales de référence. Cela se confirme à propos de deux des trois exemples de « contextes qui ont du sens » associés à la compétence $n^{\circ} 6$ :

« [1] Dans le cadre de la visite d'un musée, la lecture d'un "nouveau roman" ou la mise en scène d'une œuvre dramatique lue, les élèves sont confrontés à une œuvre consacrée qu'ils jugent illisible ou illégitime, par exemple une peinture ou une sculpture abstraite. En croisant différents éclairages (le fonctionnement social de la littérature, la littérature comme histoire des formes, la littérature comme lieu 
du jeu avec les normes...), ils sont invités à expliquer le jugement de valeur positif dont l'œuvre bénéficie. Variante : expliquer un jugement de valeur négatif du passé à propos d'une œuvre aujourd'hui appréciée et reconnue.

[2] Après la lecture d'une œuvre ou sa découverte au théâtre, les élèves abordent la question de la fonction sociale de la littérature, de l'engagement de l'écrivain ou de l'artiste (par ex., O vous, frères humains d'Albert Cohen, ou Inconnu à cette adresse de Kressmann Taylor ou encore la poésie de la résistance. Ils construisent un réseau de textes écrits, d'œuvres cinématographiques ou portées à la scène et, sous divers éclairages croisés (fonctionnement social de la littérature, réservoir de valeurs et d'idées, lieu du jeu avec les normes...), ils tentent d'élaborer une réponse tenant compte de la complexité des données.

[3] Les élèves doivent créer quelques pages d'anthologie (texte de synthèse) sur une œuvre, un auteur, un mouvement ( $c f$. fiche 2). Pour mener à bien ce travail, ils pourraient exploiter l'apport de plusieurs éclairages : la littérature comme production d'un auteur, le fonctionnement social de la littérature, la littérature comme patrimoine à transmettre et la littérature dans le champ des productions artistiques. » (p. 29)

Le réseau catholique se distingue par ailleurs en associant l'approche par compétence à la séquence didactique en trois temps. Selon son programme, tout apprentissage est en effet censé commencer par une pratique initiale - phrase de contextualisation où la production visée est réalisée à des fins diagnostiques, sans autre outil que la représentation spontanée qu'en ont les élèves -, se poursuivre par des ateliers de structuration - phase de décontextualisation où les ressources nécessaires à la réussite de la production sont « analysées », c'est-à-dire enseignées et exercées -, et s'achever par une production finale - phase de recontextualisation, dans laquelle les ressources qui viennent d'être apprises sont réinvesties dans une tâche similaire à la tâche initiale.

Enfin, contrairement au réseau de la Communauté, le réseau catholique place explicitement l'apprentissage de la lecture sous l'égide de la notion de lecture littéraire, qu'elle étaie par différentes sources (référence à des écrits de Tauveron, de Poslaniec, de votre serviteur) et qu'elle combine à l'approche par compétence en la définissant comme « une activité complexe de résolution de problème "qui tresse incessamment les démarches complémentaires” » ${ }^{(12)}$.

Dans ce programme, la compétence apparait donc moins comme une notion exclusive que comme un élément dans une combinaison : la logique des compétences est ici étroitement imbriquée à celles de la lecture littéraire et de la séquence didactique en trois temps.

(12) Programme Français $3^{e}$ degré Humanités générales et technologiques, op. cit., p. 11 ; cette définition se réfère explicitement à celle que j'ai proposée dans l'ouvrage Pour une lecture littéraire 2 (Dufays, $1996: 174)$. 


\section{Les implications de l'approche par compétence pour la didactique de la littérature}

Maintenant qu'on voit quelles activités l'approche par compétence préconise dans le cadre de l'enseignement de la lecture et de la littérature, il est intéressant de se demander dans quelle mesure celles-ci diffèrent de celles qui prévalaient auparavant.

\subsection{Une approche moins novatrice qu'il n'y parait}

A ce propos, il faut d'abord souligner, à la suite de Romainville (2001 : 208), que l'approche par compétence n'a pas le même impact sur toutes les disciplines scolaires. En effet, si une discipline comme les mathématiques éprouve des difficultés à proposer aux élèves des tâches enracinées dans des situations-problèmes, cela s'avère beaucoup moins problématique pour le français, où bon nombre de productions traditionnelles apparaissent comme des tâches complexes qui comportent déjà une finalité sociale assez évidente. L'écrit argumenté, la présentation d'exposés oraux, la recherche documentaire ont été récupérés sans difficulté par l'approche par compétence parce qu'ils lui sont très antérieurs. Dans le cas du français, la logique des compétences n'a donc pas engendré de réelle révolution.

\subsection{Une multiplication des «textes du lecteur»}

Néanmoins, à propos de la lecture et de la littérature, l'approche par compétence a modifié assez sensiblement la donne. En effet, que demandait-on généralement aux élèves pour attester leurs aptitudes dans ces domaines avant l'avènement de ce paradigme ? Pour la lecture, des réponses à des questionnaires et des analyses de texte, qui pouvaient parfois prendre la forme élaborée de commentaires composés ; pour la littérature, des questions de restitution, de synthèse ou de compréhension relatives aux matières vues par le professeur (Dufays, 2002). Comme le montrent les programmes, à partir du moment où le privilège est accordé aux "tâches complexes significatives », les enseignants sont invités à multiplier les «textes du lecteur» (Dufays, 2011), c'est-à-dire des productions écrites d'une certaine ampleur dans lesquelles le lecteur manifeste divers aspects de sa réception en s'inscrivant dans un genre social déterminé : journal de lecture, note critique, argumentation en faveur ou en défaveur d'une œuvre, débat interprétatif...

\subsection{De nouveaux genres scolaires liés à la littérature}

Une autre conséquence de la priorité accordée aux productions complexes évaluables est la distinction entre les tâches de lecture et les tâches relatives à la littérature, qu'on a pu constater notamment dans le programme du $3^{\mathrm{e}}$ degré du réseau catholique belge. On voit bien en effet que, même si elles se rejoignent souvent, ces deux séries d'activités peuvent fort bien diverger, car d'une part on peut évaluer la lecture d'autres textes que celle des textes littéraires, et d'autre 
part l'appropriation de la littérature ne passe pas seulement par la lecture. En l'occurrence, à côté des textes du lecteur évoqués ci-dessus, les programmes préconisent de nouveaux genres d'écrit scolaires spécifiquement centrés sur l'appropriation de la littérature : l'exposé oral présentant un courant ou un problème littéraire (réseau de la Communauté), le « texte de synthèse » mettant en perspective différents éclairages sur la notion de littérature, le bilan réflexif évaluant le chemin parcouru entre le début et la fin de l'apprentissage, et la mise en perspective d'une pratique ou d'un fait d'actualité littéraire (compétence $\mathrm{n}^{\circ} 6 \mathrm{du} 3^{\mathrm{e}}$ degré du réseau catholique).

\subsection{Des tâches ou des situations?}

Cela étant, comme je l'ai déjà souligné, les programmes belges révèlent une tension entre deux manières de mobiliser la compétence : d'un côté, en assimilant la performance attendue à l'effectuation d'une " tâche-problème », le réseau officiel insiste sur le rôle de l'enseignant qui définit la tâche ; de l'autre, en parlant de «situation-problème ", le réseau catholique suggère que l'identification du problème à résoudre pourrait résulter d'une négociation entre le professeur et les élèves. Une «situation », au sens où l'entendait déjà Brousseau pour la didactique des mathématiques, est en effet quelque chose qui peut être identifié et analysé collectivement, alors qu'une «tâche » est une activité qu'une personne pourvue d'une certaine autorité assigne à d'autres.

\subsection{Approche par compétence et séquence didactique en trois temps}

On a vu également qu'une autre spécificité du programme du réseau catholique est qu'il associe la notion de compétence à celle de séquence didactique en trois temps. Celle-ci cependant s'est développée parallèlement à celle-là, puisque c'est au cours des années 1990 qu'elle a fait l'objet d'une modélisation et d'une préconisation tant dans les programmes précédents du même réseau que dans les outils d'enseignement développés par les didacticiens de l'école de Genève (Dolz, Noverraz \& Schneuwly, 2001).

Cette conjugaison avec la notion de séquence ajoute à la notion de compétence un élément qui ne figurait pas dans son socle définitionnel primitif : l'appel à fonder l'apprentissage sur l'analyse de la production initiale, ce qui revient à se centrer tout autant sur l'input et sur les démarches de l'apprenant que sur la performance finale. Qui plus est, les activités de structuration, que l'approche par compétence tend à marginaliser au bénéfice des productions complexes, retrouvent dans la séquence toute leur légitimité. On peut donc dire que, dans la séquence en trois temps, l'enseignement n'est plus subordonné à la logique de la performance, mais au contraire, celle-ci est absorbée dans une logique plus puissante et plus riche qui la dépasse et en relativise l'impact.

Différence entre situation et tâche, déplacements liés à la combinaison avec la séquence en trois temps : voilà qui montre bien que l'approche par compétence est sujette à interprétations et n'impose pas en soi une logique unique aux systèmes éducatifs. Tout au plus un point commun peut-il être imputé à tous les enseignants qui se réclament de cette approche : le souci de multiplier les tâches complexes. 


\section{Quel enseignement de la lecture et de la littérature fondé sur les compétences?}

\section{1. Échos des pratiques réelles}

SI les prescriptions des programmes donnent une idée assez variée et précise de ce que requiert l'approche par compétence dans le domaine de l'enseignement-apprentissage de la lecture et de la littérature, qu'en est-il de ses réalisations sur le terrain ? On ne dispose guère, pour l'heure, d'enquêtes à grande échelle pour le vérifier, mais dans le cas de la Belgique francophone, les pratiques enseignantes font l'objet depuis près d'une décennie d'une politique de contrôle et de régulation intensive par le système éducatif (obligation pour toutes les écoles de mettre en œuvre des épreuves externes non certificatives à différents niveaux scolaires, visites accrues d'inspecteurs et de conseillers pédagogiques) qui ont forcément des effets unificateurs. Qu'ils le veuillent ou non, les enseignants sont de plus en plus tenus de rédiger leurs épreuves en termes de productions globales complexes.

Deux enquêtes modestes que j'ai moi-même réalisées ces dernières années amènent à nuancer cette tendance. La première, effectuée en 2008-2009 auprès de 36 enseignants des trois dernières années du secondaire représentatifs de la diversité des régions et des publics scolaires de la Belgique francophone ${ }^{(13)}, \mathrm{m}$ 'a permis de dresser la liste des tâches de lecture qu'ils faisaient réaliser par leurs élèves. Le constat qui en résulte est que les tâches complexes sont certes devenues dominantes par rapport aux activités " de structuration », mais qu'elles restent fortement concurrencées par les tâches décontextualisées. Voici en effet le classement des « textes du lecteur » signalés par les 36 enseignants qui ont répondu à l'enquête (chiffres présentés également dans Dufays, 2011) :

- Réponses à des questionnaires 17

- Note critique 15

- Analyse d'un roman, d'une nouvelle ou d'une pièce 12

- Exposé oral sur un livre, un auteur 12

- Fiche de lecture 10

- Mise en scène d'une pièce ou d'un extrait de théâtre 7

- Comparaison de textes, ou entre une cuvre et sa version filmique 7

- Écrit argumenté, dissertation sur un texte 7

- Lettre de motivation, lettre ouverte à l'auteur, lettre à un élève d'une autre classe 6

- Journal de lecture 5

- Analyse de poèmes 4

- Interview fictive de l'auteur ou d'un personnage 4

- Rédaction d'une autre fin ou d'une suite du texte 4

- Plaidoyer, billet d'humeur 4

(13) Il s'agissait de maitres de stage issus de toutes les régions de la Belgique francophone et des différentes filières d'enseignement (général / technique / professionnel). Le seul biais lié à cette population est qu'elle est constituée d'enseignants plutôt plus engagés dans leur métier que la moyenne puisqu'ils acceptent de consacrer une partie de leur temps à l'encadrement de stages de futurs collègues. 
- Transposition d'un texte romanesque en pièce de théâtre 3

- Production artistique (affiche, dessin) sur un livre 3

- Cercle de lecture, débat 2

- Quatrième de couverture 2

- Rédaction d'un extrait de roman en changeant de narrateur ou de point de vue 2

- Commentaire composé 1

- Procès d'un auteur, d'un livre, d'un texte 1

- Participation à un jury littéraire (prix des lycéens) 1

- Compte rendu sous forme de notice médicamenteuse 1

- Un « quizz » à propos de la lecture d'un roman historique 1

On le voit, la pratique qui l'emporte de loin et est revendiquée par près d'un professeur sur deux est le questionnaire de lecture, qui constitue la tâche de structuration par excellence. Trois points sont cependant à souligner :

$1^{\circ}$ rares sont les enseignants qui disent pratiquer cette tâche à l'exclusion des autres. Plus précisément, presque tous affirment combiner les questionnaires de lecture avec des tâches complexes comme la note critique ou l'écrit argumenté à propos d'un texte. Autrement dit, aux yeux de la majorité des enseignants interrogés, la logique des compétences n'est pas perçue comme exclusive, mais comme complémentaire à d'autres logiques ; $2^{\circ}$ si les tâches complexes envisagées par ces enseignants sont particulièrement variées, très peu d'entre elles sont pratiquées en grande quantité : on peut donc dire que la logique des compétences, quand elle est appliquée, n'a pas pour effet d'unifier les pratiques, mais seulement de les rendre plus convergentes dans leurs finalités ;

$3^{\circ}$ parmi les tâches complexes, seule la note critique ${ }^{(14)}$ émerge (avec $41 \%$ de mentions) en tant que tâche relativement nouvelle (elle n'a fait son apparition dans les programmes et dans les formations qu'au début des années 2000), tandis que le journal de lecture n'obtient que $15 \%$ des mentions. Pour le reste, les tâches complexes les mieux classées peuvent être qualifiées de traditionnelles dans la mesure où elles préexistaient à l'approche par compétence : l'analyse d'un roman, d'une nouvelle ou d'une pièce (33\%), l'exposé oral sur un livre, un auteur (33\%), la fiche de lecture $(28 \%)$, la mise en scène d'une pièce ou d'un extrait de théâtre $(19 \%)$, la comparaison de textes, ou entre une œuvre et sa version filmique (19\%), ou encore l'écrit argumenté ou la dissertation sur un texte (19\%).

Une autre micro-enquête, menée en janvier 2007, m'a permis de recueillir dans trois écoles choisies pour leurs profils contrastés un ensemble de pratiques évaluatives relatives à une session d'examens. Mon but, à nouveau, était de voir dans quelle mesure ces pratiques laissaient une place aux tâches globales et, plus généralement, s'inscrivaient dans l'approche par compétence. Ici encore, l'enquête révèle une situation contrastée : si tous les enseignants articulent explici-

(14) Le programme du $2^{\mathrm{e}}$ degré de la Fésec la définit comme suit : « texte bref qui vise à persuader ou à dissuader le destinataire de s'engager à son tour dans la découverte d'une œuvre. La note critique est un texte composite qui comporte à la fois une partie informative (éléments matériels sur le contexte de diffusion, bref résumé ou description du contenu) et une partie argumentative (appréciation personnelle argumentée sur divers aspects de l'œuvre) » (Programme Français $2^{e}$ degré, op. cit., p. 30). 
tement leurs questions d'examens à des compétences du programme, certains se montrent sélectifs, ne retenant que trois compétences sur les six prescrites, d'autres n'hésitent pas à l'inverse à interroger leurs élèves sur des compétences absentes du programmes ou prescrites pour un autre degré d'enseignement, et enfin, si tous amènent bien leurs élèves à effectuer des tâches complexes, celles-ci sont proposées en combinaison avec des questions de pure restitution, et leur degré de complexité, pour un même niveau scolaire, varie parfois considérablement.

Cela étant, l'écho le plus objectif dont on dispose actuellement à propos des pratiques effectives est celui qui émane des évaluations externes. Certes, cellesci, qui sont imposées par le système éducatif, ne reflètent guère les pratiques enseignantes « ordinaires "; néanmoins, l'évolution de leurs résultats au fil des années permet de déceler si les activités proposées dans ce cadre paraissent progressivement mieux maitrisées par les élèves - et donc davantage pratiquées par les enseignants - ou non. Et sur ce point, les résultats sont éclairants : en Belgique francophone, l'évaluation externe la plus emblématique, celle qui est réalisée tous les trois ans dans le cadre de Pisa - et qui est «relayée » par des épreuves externes propres au système éducatif belge - a connu en 2010 une progression assez significative en ce qui concerne la maitrise des tâches complexes de lecture fonctionnelle. Les responsables de l'institution scolaire belge s'en sont réjouis, soulignant qu'il y avait là un indice du succès de la politique de formation ciblée sur les compétences qui était mise en œuvre depuis une dizaine d'année. Même si cela ne prouve en rien que les enseignants font massivement pratiquer les épreuves de ce type par leurs élèves, cela indique du moins qu'elles sont entrées dans la culture des écoles et font partie désormais des tâches auxquelles les élèves sont préparés. Il faut toutefois souligner que ces épreuves, qui sont rédigées selon des standards internationaux, concernent presque exclusivement la lecture de textes fonctionnels (articles descriptifs, informatifs ou argumentatifs) : il est donc impossible d'en tirer quelque conclusion que ce soit à propos de l'enseignement de la littérature.

\subsection{Bilan critique}

Si les pages qui précèdent se sont limitées - par souci de réalisme méthodologique - à évoquer le seul contexte de la Belgique francophone, l'analyse de la variété des usages qui y sont faits de l'approche par compétence autorise maintenant, me semble-t-il, à tirer quelques éléments de réponse à la question qui a suscité cette investigation, à savoir : quelle est la pertinence de l'approche par compétence pour l'apprentissage de la lecture et de la littérature ?

Je pointerai d'abord ici six éléments qui me semblent problématiques.

$1^{\circ} \mathrm{Si}$ l'approche par compétence favorise sans conteste la mise en activité des élèves et le développement de productions complexes liées à la lecture et à la littérature, sa productivité ne suffit pas à garantir sa qualité : les productions complexes risquent de demeurer superficielles, voire vaines, si elle ne sont pas articulées d'une part à un modèle riche du rapport à la littérature comme celui de la lecture littéraire en tant qu'activité dialectique et d'autre part à des seuils de complexité progressifs qui incitent les élèves à devenir plus compétents d'année en année. Or l'analyse de ces seuils n'en est, aujourd'hui qu'à ses premiers bal- 
butiements. La progression curriculaire des apprentissages suscite depuis peu un intérêt croissant des didacticiens ${ }^{(15)}$, mais il faudra sans doute attendre plusieurs années avant qu'il n'en sorte un cadre de référence convaincant comparable à celui qui a été élaboré pour le français langue étrangère par le Conseil de l'Europe.

$2^{\circ} \mathrm{Vu}$ que le sens, le caractère « significatif» d'un fait n'est jamais un élément objectif dont on puisse garantir la perception commune, l'appel à ancrer les compétences de lecture dans une "situation-problème significative » apparait comme une formule creuse. A titre d'exemple, le décret « Missions » déjà cité distingue trois sources de «sens » : l'épanouissement personnel, l'intégration active dans la société économique et la citoyenneté responsable. Grootaers (1999), qui relie ces trois sources aux catégories de la fidélité à soi-même, de l'utilité sociale et de l'appartenance, précise en outre que chacune d'elles prend une portée différente selon qu' on la considère à court terme, à moyen terme ou à long terme. Certes, les enseignants sont invités à poursuivre ces différentes finalités de manière complémentaire, mais chaque activité ne peut en viser qu'un nombre limité, et qui peut garantir que celles-ci feront sens aux yeux de tous les élèves? Sans doute les activités interactives et créatives tournées vers des pratiques sociales de référence plairont-elles à bon nombre d'entre eux, mais rien n'interdit de penser que d'autres trouveront davantage leur compte dans des tâches traditionnelles ou dans des cours magistraux donnés avec brio. Parce qu'il existe des conceptions très distinctes du sens d'une lecture ou d'un travail, il semble vain, en tout cas, de vouloir définir a priori le caractère significatif d'une activité littéraire. Au lieu d'inviter l'enseignant à rendre ses tâches « signifiantes », ne serait-il pas plus adéquat de l'inviter à diversifier autant que possible les sources de motivation, lesquelles comportent un ensemble de paramètres bien plus larges que ceux qui concernent la seule nature des tâches ( $c f$. notamment Viau, 1994) ?

$3^{\circ}$ Parce que, dans la logique des compétences, les savoirs sont présentés seulement comme des « ressources », dans le cas de la littérature, cette logique a amené à surdévelopper les savoir-faire, les activités de recherche, d'exposé, de synthèse, etc., au détriment de la transmission de savoirs historiques, culturels et théoriques. Ecoutons à ce propos José Soblet, secrétaire de la Fédération de l'enseignement secondaire catholique de Belgique francophone :

« Les savoirs visés sont [...] quasi exclusivement instrumentaux, les savoirs gratuits, les savoirs de mémoire, les savoirs culturels sont réputés être, je reprends des termes de l'époque, des "savoirs morts". On a été trop loin. Les plus ardents défenseurs du "tout pour et par les compétences" ont eu l'élégance de le reconnaitre, mais de façon peut-être un peu plus discrète que lors de leur tonitruante entrée en scène. On revient aujourd'hui, me semble-t-il, à un meilleur équilibre en la matière, replaçant ces compétences plutôt dans le champ des objectifs que dans celui des méthodes. Une sorte de consensus s'établit donc autour de l'éternelle dialectique entre les tenants d'un constructivisme radical et les partisans d'un équilibre basé sur la diversification des approches. » (Soblet, $2010: 31$ )

(15) C'était le thème du XI ${ }^{e}$ colloque de l'AIRDF qui s'est tenu à Liège en aout 2010. A propos de la progression des apprentissages en lecture et en littérature, voir notamment Dufays, $2010 \mathrm{~b}$ et Dufays, à paraitre. 
Cette critique est d'autant plus intéressante qu'elle émane du responsable d'une institution dont les programmes continuent à promouvoir l'approche par compétence : elle montre assez à quel point la logique des compétences a atteint ses limites et commence aujourd'hui à refluer.

$4^{\circ}$ Parce qu'elle favorise la mobilisation de ressources variées et la capacité de résoudre des problèmes complexes, la logique des compétences combat certes l'émiettement des apprentissages, comme l'écrit François Quet (2009), mais il n'est pas sûr que cette valorisation de la complexité soit propice à la motivation et à la réussite du plus grand nombre. Au contraire, Jean-Maurice Rosier a souligné que cette logique profitait surtout aux élèves les plus forts et ne manquait pas d'accroitre les échecs chez les autres :

«À long terme, cette nouvelle conception de l'évaluation en français, à base de culture praxéologique dont le fondement est le faire, la compétence en acte amènera l'amenuisement des contrôles sur les connaissances déclaratives et les interrogations sur des savoirs de restitution ou prétendus tels comme l'étude de l'histoire littéraire. Cette réduction, il faut y prendre garde, ne profitera pas aux élèves en difficulté qui se rassurent dans le répétitif et avec des stratégies moins globales que l'évaluation formative ne prétend pas cerner. Soit, le développement des compétences est consubstantiel au cours de français, mais fallait-il pour cela mépriser les activités modestes du pas à pas et ne privilégier que la logique des compétences intégratives [...] ?» (Rosier, $2002: 94-95)$.

$5^{\circ}$ Par ailleurs, on ne peut pas ignorer le fait que la logique des compétences requiert un travail de production incessant de la part des élèves et un travail d'évaluation considérable de la part de l'enseignant - car, si l'on en croit les pédagogues, une compétence ne pourrait être évaluée de manière certificative qu'après trois performances intermédiaires. De nombreux élèves, mais aussi d'enseignants se plaignent ainsi, non sans raison, d'être soumis à un régime d'évaluation permanente qui, paradoxalement, fait obstacle aux conditions élémentaires d'un bon apprentissage.

$6^{\circ}$ Enfin, plus fondamentalement, il importe de souligner qu'en matière de lecture et de littérature, l'essentiel n'est peut-être pas tant de devenir « compétent » que de trouver du plaisir et de l'intérêt à lire et à s'approprier les textes et les savoirs qui les concernent. Autrement dit, dans ce domaine, la compétence n'est pas tout, et ce serait un contresens que d'évaluer les résultats de l'enseignement de la littérature sur la seule base des productions complexes.

Ces remarques ne visent pas à rejeter l'approche par compétence mais à souligner qu'elle ne peut être, comme le dit Dumortier (2009), qu'un moyen - et j'ajouterai : un moyen parmi d'autres - d'améliorer les apprentissages des élèves. Et en tant que moyen, elle constitue moins un aboutissement qu'un chantier, encore largement à développer.

L'approfondissement critique de la logique des compétences parait d'autant plus nécessaire que cette approche comporte des enjeux non négligeables. En effet, outre qu'elle se présente à bien des égards comme plus rationnelle et plus cohérente que les autres approches, Romainville lui attribue trois atouts décisifs : $1^{\circ}$ elle a « résolument placé la mobilisation des savoirs au centre des préoccupations de l'école », $2^{\circ}$ elle « a permis à notre système scolaire de disposer, pour la 
première fois, de définitions standardisées des niveaux d'études attendus aux différents paliers de la scolarité obligatoire $», 3^{\circ}$ elle a « mis en route $[\ldots]$ un processus salutaire que l'on pourrait qualifier de "dédisciplinarisation" de l'école obligatoire » (Romainville : $2006: 26$ ).

La conclusion se doit dès lors d'être tout aussi balancée que le bilan qui la précède : si l'objectif du cours de français est réellement de former de meilleurs lecteurs et de meilleurs amateurs de littérature, il semble indispensable à la fois de proposer aux élèves des tâches complexes et de ne pas limiter l'enseignement de la lecture/littérature à la visée de « compétences ». Car se former à la lecture et à la littérature, ce n'est pas seulement devenir un interprète performant : c'est aussi vivre des expériences et acquérir des savoirs qui n'ont d'autre valeur que de nourrir notre imaginaire et de mettre le monde et nous-mêmes en perspective.

\section{Bibliographie}

BERNIÉ, Jean-Paul (2002) : «L'approche des pratiques langagières scolaires à travers la notion de "communauté discursive" : un apport à la didactique comparée ? », in Revue française de pédagogie, n¹41, oct.-nov.-déc. 2002, pp. 77-88.

BlAnCHOT, Maurice (1955) : L'Espace littéraire, Paris, Gallimard (coll. Idées, 1982).

CEPEC, sous la dir. de GILLET, Pierre (1992) : Construire la formation. Outils pour les enseignants et les formateurs, Paris, ESF, 158 p.

CRAHAY Marcel (2006) : «Dangers, incertitudes et incomplétude de la logique de la compétence en éducation », in La Revue française de pédagogie, $\mathrm{n}^{\circ} 154$ - La construction des politiques d'éducation : de nouveaux rapports entre science et politique, pp. 97-110.

DAUNAY, Bertrand (1999) : «La lecture littéraire : les risques d'une mystification », in Recherches, 30, pp. 29-59.

- (2002) : Éloge de la paraphrase, Vincennes, Presses universitaires de Vincennes.

De Certeau, Michel (1980) : L'invention du quotidien. 1. Arts de faire, Paris, Gallimard.

Dolz, Joaquim \& OLLAGNIER, Edmée (dir.) (2000) : L'énigme de la compétence en éducation, Bruxelles, De Boeck, Coll. Raisons Éducatives.

Dolz, Joaquim, NoverRAZ, Michèle et SCHNEUWLY, Bernard (2001) : S'exprimer en français : séquences didactiques pour l'oral et pour l'écrit, Bruxelles, De Boeck, 8 vol. 
DUFAYS, Jean-Louis (à paraitre) : «Lire au fil des ans. Quelle continuité des apprentissages en lecture et en littérature dans l'enseignement secondaire de transition en Belgique francophone? », in Jean-Louis Dumortier (dir.), Quelles progressions curriculaires en français? Actes du XI colloque de l'AIRDF, Namur, AIRDF.

- (2011): «Les textes du lecteur en situation scolaire », in Catherine MAZAURIC \& Gérard LANGLADE (dir.), Textes de lecteurs en formation, Berne, Peter Lang (ThéoCrit'), pp. 6-14.

- (2010a) : Stéréotype et lecture. Essai sur la réception littéraire, Berne, Peter Lang (ThéoCrit') ( $1{ }^{\text {re }}$ éd. : 1994).

- (2010b) : «Discontinuités dans l'enseignement de la littérature en Belgique francophone », in Le français aujourd'hui, 168, pp. 33-42.

- (2002) : «L'évaluation au cours de français langue première dans l'enseignement secondaire », in Léopold Paquay, Ghislain Carlier, Luc Collès, Anne-Marie Huynen (dir.), L'évaluation des compétences chez l'apprenant. Pratiques, méthodes et fondements. Actes du colloque du 22 novembre 2000, Presses universitaires de Louvain, 2002 (Recherche en formation des enseignants et en didactique), pp. 51-60.

- (1996) : «Culture/compétence/plaisir : la nécessaire alchimie de la lecture littéraire », in Jean-Louis DUfAYs, Louis GEMENNE \& Dominique LEDUR (dir.), Pour une lecture littéraire, 2. Bilan et confrontations, Bruxelles, De Boeck-Duculot.

Dufays, Jean-Louis, Gemenne, Louis \& LeDUR, Dominique (2005) : Pour une lecture littéraire. Histoire, théories, pistes pour la classe, Bruxelles, De Boeck-Duculot (Savoirs en pratique) ( $1^{\text {re }}$ éd. : 1996, sous le titre Pour une lecture littéraire 1).

DUMORTIER, Jean-Louis (2009) : «Formation littéraire et compétences. Notes pour un débat initié par l'INRP », document publié en ligne, http://litterature.inrp.fr/litterature/ discussions/enseignement-de-la-litterature-12019, consulté le $1^{\text {er }}$ mars 2011.

FORQUIN, Jean-Claude (1989) : École et culture. Le point de vue des sociologues britanniques, Bruxelles, De Boeck.

GenetTe, Gérard (1997) : L'œuvre de l'art. 2. La relation esthétique, Paris, Seuil (Poétique).

GERVAIS, Bertrand (1993) : À l'écoute de la lecture, Montréal, VLB éditeur.

GROOTAERS, Dominique (2000) : «Les ressorts de la motivation des élèves », in Exposant neuf, $\mathrm{n}^{\circ} 1$, pp. 13-17.

ISER, Wolfgang (1985) : L'acte de lecture. Théorie de l'effet esthétique, Liège, Mardaga (Philosophie et langage) (éd. orig. : 1976).

JOUVE, Vincent (1993) : La lecture, Paris, Hachette.

MARGHESCOU, Mircea (2009) : Le concept de littérarité. Critique de la métalittérature, Paris, Kimé ( $1^{\text {re }}$ éd. : 1974).

MICHEL, Raymond (1998) : «Lecture méthodique ou méthode de lecture à l'usage des élèves de lycée : un objet didactique non identifié », Pratiques, ${ }^{\circ}$ 97-98, pp. 59-103.

- (2001) : « Lecture cursive et lecture analytique. Du goût et du plaisir de lire », Pratiques, $\mathrm{n}^{\circ}$ 109/110, pp. 179-205. 
PERRENOUD, Philippe (2000) : «Construire des compétences », entretien recueilli par Paola Gentile et Roberta Bencini [texte original paru en portugais dans Nova Escola (Brésil), 2000, pp. 19-31], http://www.unige.ch/fapse/SSE/teachers/perrenoud/php_main/ php_2000/2000_30. html, consulté le 28/12/2010.

PICARD, Michel (1986) : La lecture comme jeu, Paris, Minuit.

Poslaniec, Christian (2002) : Vous avez dit «littérature »?, Paris, Hachette.

QUET, François (2009) : «Introduction» à la discussion en ligne Enseignement de la littérature : L'approche par compétences a-t-elle un sens?, document publié en ligne, http ://litterature.inrp.fr/litterature/discussions/enseignement-de-la-litterature-12019, consulté le $1^{\mathrm{er}}$ mars 2011.

REY, Bernard (1998) : Les compétences transversales en question, Bruxelles, De Boeck.

Riffaterre, Michael (1979): La production du texte, Paris, Seuil (Poétique).

ROEGIERS, Xavier (2000) : Une pédagogie de l'intégration. Compétences et intégration des acquis dans l'enseignement, Bruxelles, De Boeck.

ROMAINVILLE, Marc (2001) : «Les implications didactiques de l'approche par compétences », Enjeux, n ${ }^{\circ}$ 1, pp. 199-223.

- (2006) : «L'approche par compétences en Belgique francophone : où en est-on? », Les cahiers pédagogiques, ${ }^{\circ} 439$, pp. 24-25.

- (2008) : «Et si on arrêtait de tirer sur les compétences? », Indirect, 2008, pp. 31-43.

ROSIER, Jean-Maurice (2002) : La didactique du français, Paris, P.U.F. (Que saisje ?).

ROUXEL, Annie \& LANGLADE, Gérard (dir.) (2004) : Le sujet lecteur. Lecture subjective et enseignement de la littérature, Rennes, P.U.R.

Simard, Claude, DufaYs, Jean-Louis, Dolz, Joaquim \& GarCia-Debanc, Claudine (2010) : Didactique du français langue première, Bruxelles, De Boeck, $459 \mathrm{p}$.

SOBLET, José (2010) : «Les programmes du troisième degré de transition de l'enseignement secondaire catholique ", in Jean-Louis DUfAYs, Marie-Laurence DE KEERSMAECKER \& Alain MEURANT (dir.), Quelles pratiques didactiques pour favoriser la transition secondaire-université? Gros plan sur les programmes du $3^{e}$ degré de l'enseignement secondaire de transition, Louvain-la-Neuve : Presses universitaires de Louvain (Recherches en formation des enseignants et en didactique), pp. 29-35.

STEINMETZ, Horst (1981) : "Réception et interprétation », in Kibedi VARGA, Aron (dir.), Théorie de la littérature, Paris, Picard, pp. 193-209.

STIERLE, Karlheinz (1979) : «Réception et fiction», in Poétique, 39, pp. 299-320.

VIAU, Rolland (1994) : La motivation en contexte scolaire, Bruxelles, De Boeck (Pratiques pédagogiques). 